\title{
NEAR REAL-TIME GEOREFERENCE OF UMANNED AERIAL VEHICLE IMAGES FOR POST-EARTHQUAKE RESPONSE
}

\author{
Wang Shumin*, Wang Xiaoqing, Dou Aixia, Yuan Xiaoxiang, Ding Ling, Ding Xiang \\ Institute of Earthquake Forecasting, China Earthquake Administration, Beijing, China-(wmenu, wangxiaoq517, axdothy, \\ yuanxx1983, dingxiang525)@163.com; xiaodingdj@126.com
}

\author{
Commission III, ICWG III/Iva
}

KEY WORDS: UAV images, Georeference, Earthquake emergency, Disaster information, World File

\begin{abstract}
:
The rapid collection of Unmanned Aerial Vehicle (UAV) remote sensing images plays an important role in the fast submitting disaster information and the monitored serious damaged objects after the earthquake. However, for hundreds of UAV images collected in one flight sortie, the traditional data processing methods are image stitching and three-dimensional reconstruction, which take one to several hours, and affect the speed of disaster response. If the manual searching method is employed, we will spend much more time to select the images and the find images do not have spatial reference. Therefore, a near-real-time rapid georeference method for UAV remote sensing disaster data is proposed in this paper. The UAV images are achieved georeference combined with the position and attitude data collected by UAV flight control system, and the georeferenced data is organized by means of world file which is developed by ESRI. The C \# language is adopted to compile the UAV images rapid georeference software, combined with Geospatial Data Abstraction Library (GDAL). The result shows that it can realize rapid georeference of remote sensing disaster images for up to one thousand UAV images within one minute, and meets the demand of rapid disaster response, which is of great value in disaster emergency application.
\end{abstract}

\section{INTRODUCTION}

The high resolution remote sensing images collected based on the UAV system has become an important technical mean to quickly access and view the disaster information, and attracts much more attention recently(Aicardi et al., 2014; Baiocchi et al., 2013; Choi et al., 2011). In most instances, the collected UAV remote sensing images are stitched to make panoramic image through the commercial software such as PhotoScan, or reconstructed the 3D point cloud model by means of Smart3D software. All of these methods need features extraction, features matching, bundle adjustment and so on, which take much time to generate the image map(Tanathong et al., 2011; Turner et al., 2014; Zhou et al., 2009). In the period of postearthquake response, rapid submitting the damaged area is very important to rescue people in the damaged buildings at the first time and commence the flowing work. Considering the speed of the UAV images georeference, we design and develop the rapid georeference algorithm. Firstly, we deduce the rapid georeference model based on the UAV images collected system, and adopt the world file to organize the georeferenced image data, which designed and developed by ESRI. It avoids the image pixels resampling and saves much time. We can get the georeference parameters from the UAV rapid geographical reference model, which are needed in the world file. Secondly, the $\mathrm{C \#}$ language is employed to compile the rapid geographical reference software, combined with the GDAL library. It can process all the UAV images. Finally, we use the UAV images collected in Danlin county, Sichuan province to validate the robustness of the algorithm and the rapid georeference software. The result indicates that it can achieve the UAV images georeference within one minute for up to 1000 images. It satisfied the need of post-earthquake response.

\section{RAPID GEOREFERENCE MODEL AND DATA ORGANIZATION}

\subsection{Rapid Georeference Model}

UAV remote sensing images collection system is usually equipped with high-resolution digital camera and automatic pilot system (Nedjati et al., 2016), which controls the aircraft flight and camera exposure, and records position and attitude data at the time of camera exposes. Since the automatic pilot system and digital camera are installed tightly, we can think the automatic pilot system installed along the optical axis of the digital camera theoretically. And the position and attitude data collected from the automatic pilot system indicates the digital camera position and the attitude change at the time of camera exposure. Therefore, the rapid georeference model can express as follows:

$$
\left[\begin{array}{l}
x^{\prime} \\
y^{\prime}
\end{array}\right]=\lambda\left[\begin{array}{r}
\cos \theta, \sin \theta \\
-\sin \theta, \cos \theta
\end{array}\right]\left[\begin{array}{l}
x \\
y
\end{array}\right]+\left[\begin{array}{l}
T x \\
T y
\end{array}\right]
$$

Where $\left[x^{\prime}, y^{\prime}\right]^{T}=$ coordinates in geospatial reference system

$$
\begin{aligned}
& {[x, y]^{\mathrm{T}}=\text { pixel coordinates in the raw image space }} \\
& \lambda=\text { scale factor } \\
& \theta=\text { rotation angle } \\
& T x=\text { translation size along the } \mathrm{x} \text { axis } \\
& T y=\text { translation size along the } \mathrm{y} \text { axis }
\end{aligned}
$$

In the formula (1), it shows the UAV image rapid georeference mode consists of image scale, rotation and image translation. It indicates the attitude and location of camera changes accompanying with the UAV platform. 


\subsection{Data Organization}

In order to improve the speed of UAV images geographical reference and save time, we don't implement the images resampling in the georeference steps. We adopt the World File to organize the georeferenced UAV image data, which is developed by ESRI company, and need six parameters to achieve geographical reference. The parameters are organized in a file with the specified suffix name and text encoding, which enables the Geographic Information System (GIS) to automatically realize the georeferenced raster image data. These six parameters also constitute an affine transform matrix as follows:

$$
\left[\begin{array}{l}
x^{\prime} \\
y^{\prime}
\end{array}\right]=\left[\begin{array}{lll}
A & B & C \\
D & E & F
\end{array}\right]\left[\begin{array}{l}
x \\
y \\
1
\end{array}\right]
$$

Where $\left[x^{\prime}, y^{\prime}\right]^{T}=$ coordinates in geospatial reference system

$[x, y]^{\mathrm{T}}=$ pixel coordinates in image reference system

$\mathrm{A}=$ scale factor on the $\mathrm{x}$ axis

$\mathrm{E}=$ scale factor on the $\mathrm{y}$ axis

$\mathrm{B}, \mathrm{D}=$ rotation factors

$\mathrm{C}, \mathrm{F}=$ coordinates of image up-left corner

In the formula (2), all the coefficients need could be deduced from formula (1).

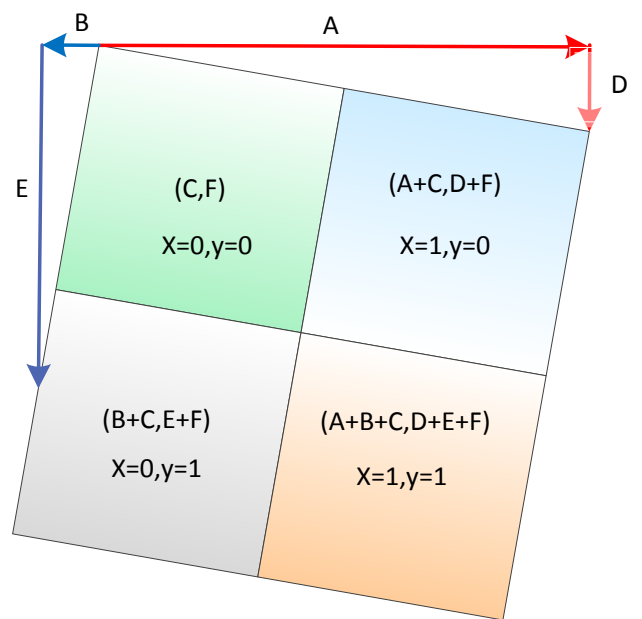

Figure1. The image georeferenced based on the world file

In the figure 2, it shows the image pixels get the georeference coordinates. When $\mathrm{x}$ equals 0 , and $\mathrm{y}$ equals 0 , the georeferenced image pixel $x^{\prime}$ equals $\mathrm{C}$, and $y^{\prime}$ equals $\mathrm{F}$. When $\mathrm{x}$ equals 1 , and $\mathrm{y}$ equals 1 , the georeferenced image pixel $x^{\prime}$ equals $\mathrm{A}+\mathrm{B}+\mathrm{C}$, and $y^{\prime}$ equals $\mathrm{D}+\mathrm{E}+\mathrm{F}$. All the image pixels could be calculated the georeferenced coordinates from formula (2). In the formula (2), the different coefficients could bring different image transformations. If we adopt the affine transformation, the image will be distortion after georeference. And if we use the image rigid body transformation, there are just scale, translation and rotation transformation. It could not cause the image distortion. In this paper, we adopt the image rigid body transformation to carry out the image spatial transformation.

\section{EXPERIMENTS AND ANALYSIS}

In the experiment, we use a fixed-wing UAV to collect the image data in Danling County, Sichuan Province. The fixedwing UAV is equipped with a Sony DSC-RX1R II digital camera. The camera focal length is $20 \mathrm{~mm}$, the CCD size is $23.5 \times 15.6 \mathrm{~mm}$, and the image captured resolution is $7952 \times 5304$ UAV flew height is average $250 \mathrm{~m}, 1025$ images are captured, and the total amount of data set is up to 18GB. We design and develop the software and run the software on IBM T430 laptop computer, equipped with Intel Core i5 2.60GHz CPU and 4GB memory.

The figure 2 shows the software interface. In the software interface, we need to input the position and attitudes data, UAV images, camera parameters and the flight parameters. After all data needed input into the software, it can achieve the UAV images georeference automatically. It also can generate the outline of the georeferenced UAV images with vector file. It could be overlaid with the reference image, which could be used for selecting the suitable UAV image to submit. It is very simple and convenient for post-earthquake response.

All images are achieved rapid geographical reference within one minute, as figure 3 . From the georeferenced images, we can know the objects distribution on the ground surface, and select the images on which there are objects we are interest to submit. We could know the roads going, the playing ground position and the buildings distribution. It is very useful in the early earthquake emergency period. For example, we can get the building status before and after the earthquake from the google image and the rapid georeferenced image in the figure 4 although there is some gap between the georeferenced image and the google image, it is acceptable and very useful in the earthquake response. 
The International Archives of the Photogrammetry, Remote Sensing and Spatial Information Sciences, Volume XLII-3, 2018 ISPRS TC III Mid-term Symposium "Developments, Technologies and Applications in Remote Sensing”, 7-10 May, Beijing, China

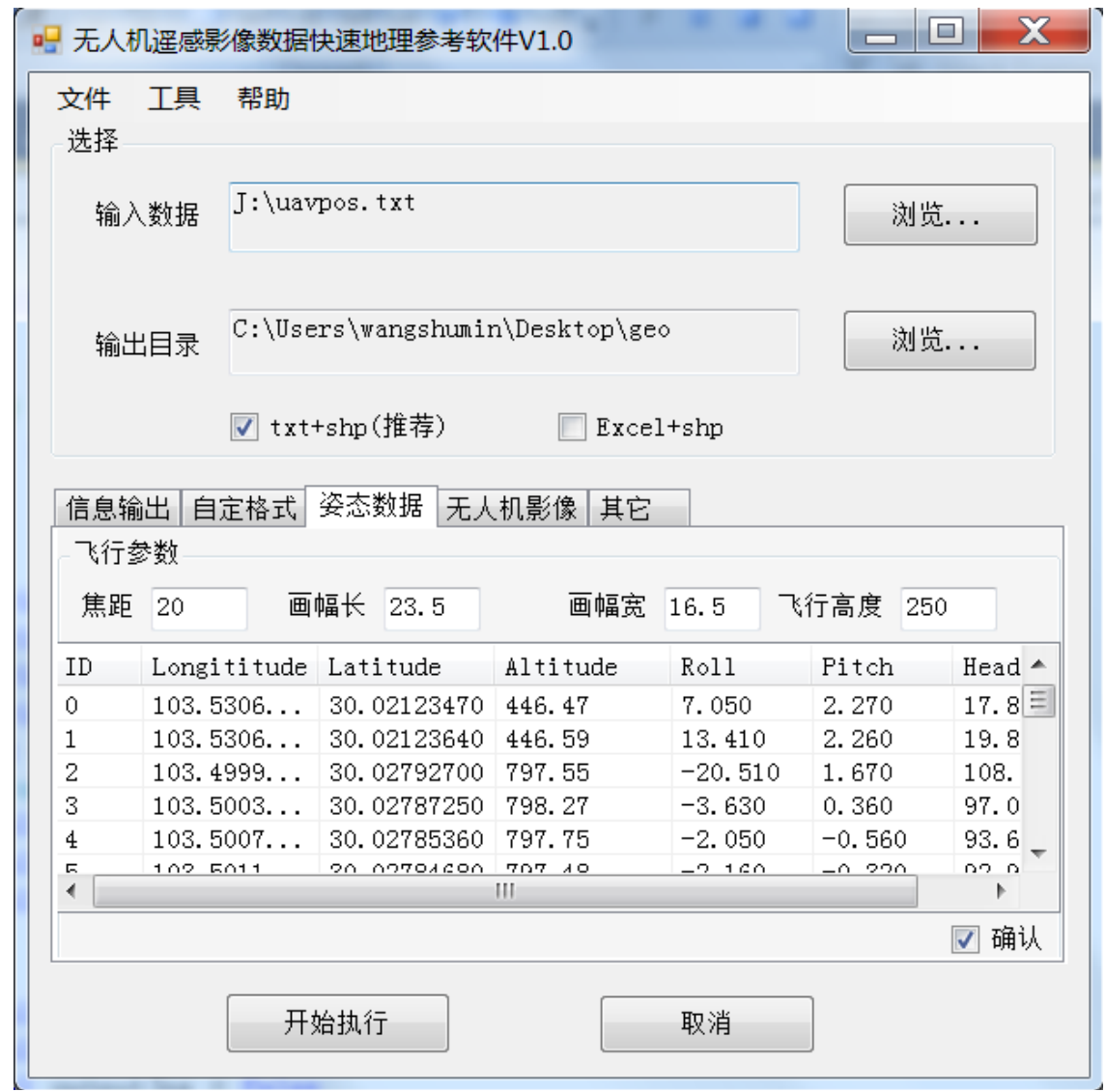

Figure. 2 The interface of the rapid geographical reference software

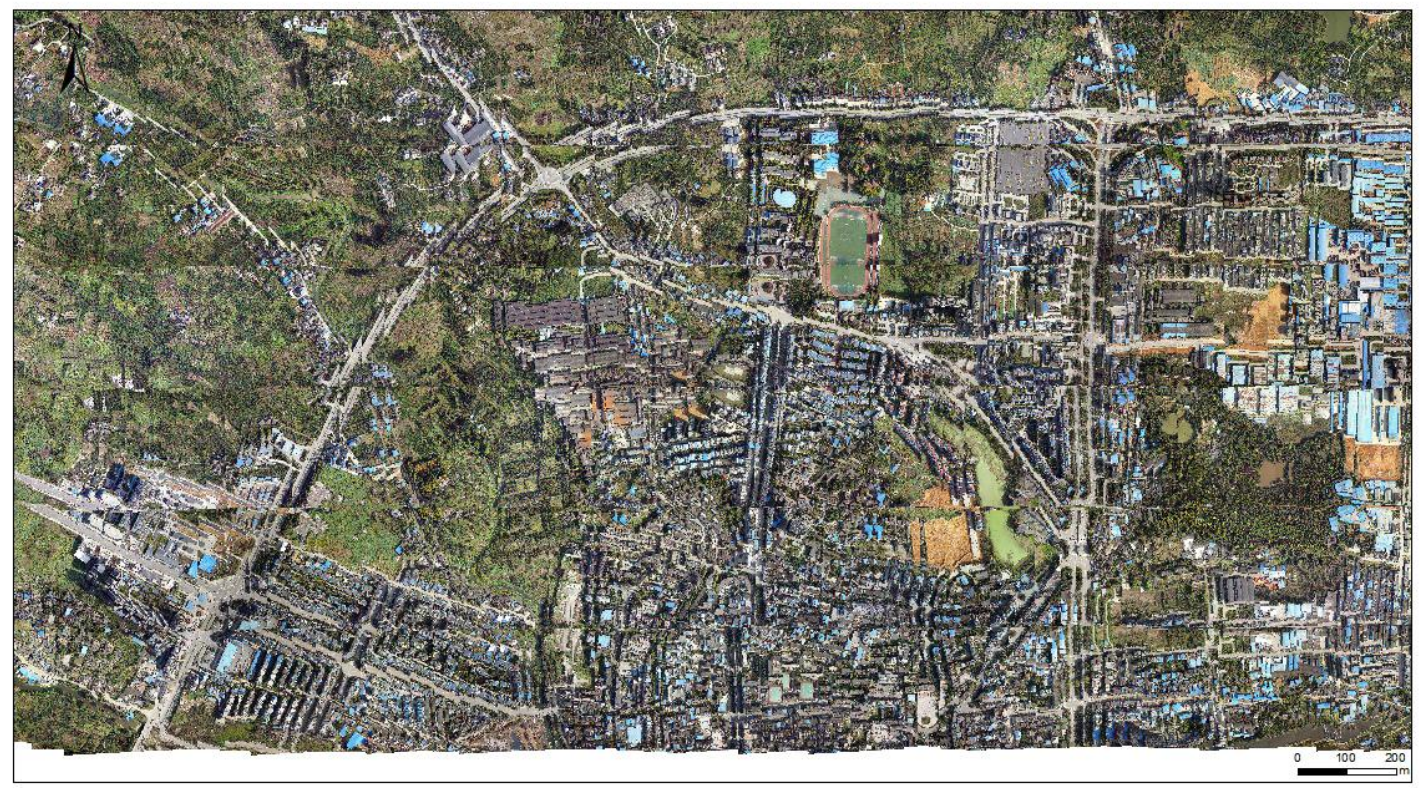

Figure. 3 The result of UAV images rapid geographical reference 


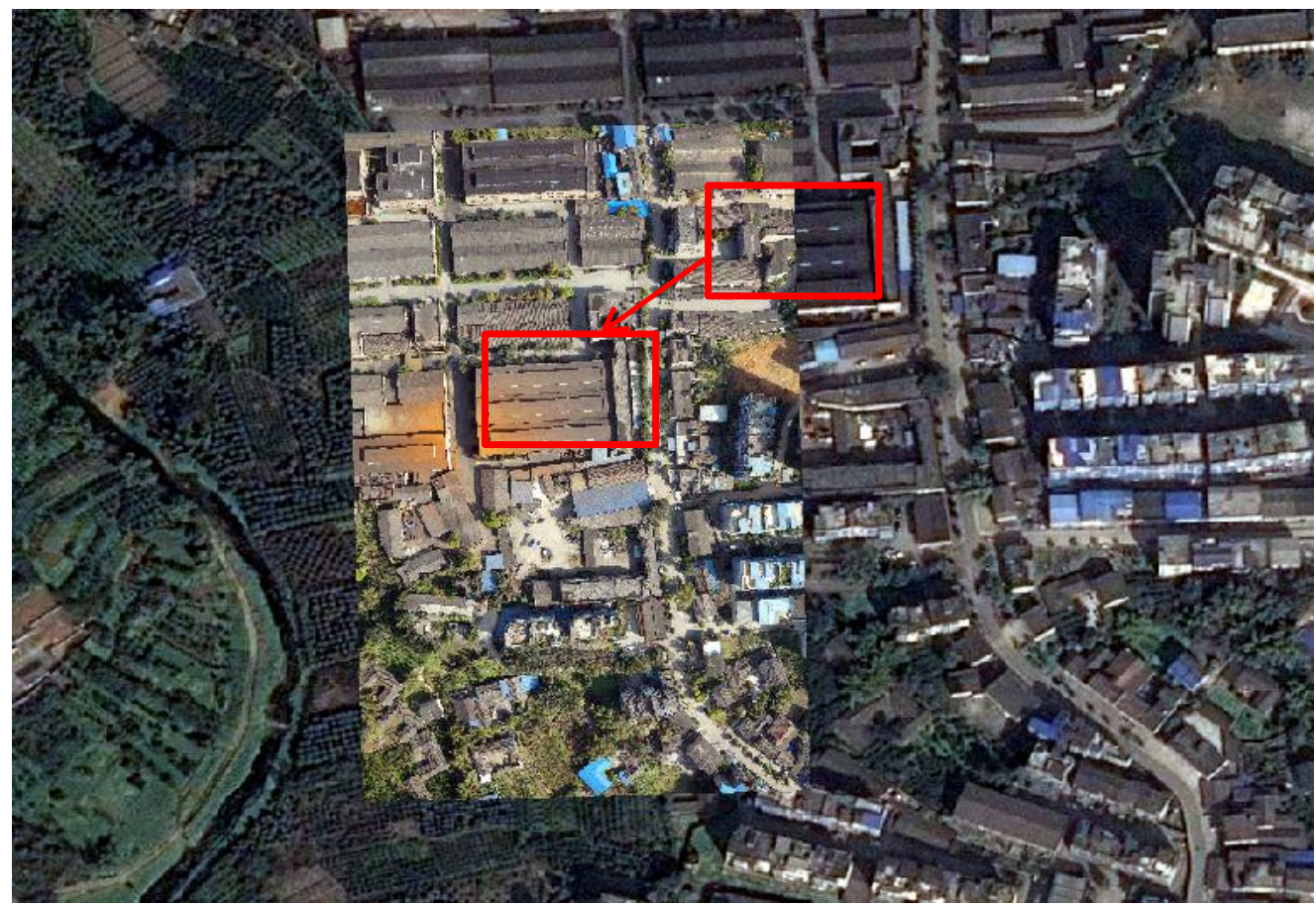

Figure 4. The georeferenced image overlaid on the google image

\section{CONCLUSION}

In this paper, we discuss the UAV images rapid Geographical reference methods, and design and develop the rapid georeference software. All the images could be georeferenced in very short time, and the georeferenced accuracy is dependent on accuracy of position and attitude parameters output from the autopilot system. It is acceptable in the earthquake emergency period. Therefore, we will attempt to install the DGPS on the UAV to improve the positioning accuracy, and study the methods of improve the georeference accuracy based on the google image.

\section{ACKNOWLEDGEMENTS}

This work funded by National Key Research and Development Program of China (2017YFB0504104) and the Fundamental Research Funds of the Institute of Earthquake Forecasting, China Earthquake Administration (No.2015IES0203).

\section{REFERENCES}

Aicardi, I., Chiabrando, F., Lingua, A. M., Noardo, F., Piras, M., 2014. Unmanned aerial systems for data acquisitions in disaster management applications. JUNCO, pp. 164-171.

Baiocchi, V., Dominici, D., Mormile, M., 2013. UAV application in post-seismic environment. Int. Arch. Photogramm. Remote Sens. Spatial Inf. Sci., XL-1 W(2), pp. 21-25.

Choi, K., Lee, 2011. A UAV-based close-range rapid aerial monitoring system for emergency responses. Int. Arch. Photogramm. Remote Sens. Spat. Inf. Sci., (38), pp. 247-252.

Nedjati, A., Vizvari, B., Izbirak, G., 2016. Post-earthquake response by small UAV helicopters. Natural Hazards, 80(3), pp. 1669-1688.
Tanathong, S., Lee, I., 2011. An automated real-time image georeferencing system. In Proc. Int. Conf. on Image Processing, Computer Vision and Pattern Recognition, pp. 68-74.

Turner, D., Lucieer, A., Wallace, L., 2014. Direct georeferencing of ultrahigh-resolution UAV imagery. IEEE Transactions on Geoscience and Remote Sensing, 52(5), pp. 2738-2745

Zhou, G., 2009. Near real-time orthorectification and mosaic of small UAV video flow for time-critical event response. IEEE Transactions on Geoscience and Remote Sensing, 47(3), pp. 739-747. 